\title{
Ranking products through online reviews: A method based on sentiment analysis technique and intuitionistic fuzzy set theory
}

\author{
Yang Liu, Jian-Wu Bi, Zhi-Ping Fan ${ }^{\text {a, b, * }}$ \\ ${ }^{a}$ Department of Information Management and Decision Sciences, School of Business \\ Administration, Northeastern University, Shenyang 110167, China \\ ${ }^{b}$ State Key Laboratory of Synthetical Automation for Process Industries, Northeastern \\ University, Shenyang 110819, China
}

\begin{abstract}
Online product reviews have significant impacts on consumers' purchase decisions. To support consumers' purchase decisions, how to rank the products through online reviews is a valuable research topic, while research concerning this issue is still relatively scarce. This paper proposes a method based on the sentiment analysis technique and the intuitionistic fuzzy set theory to rank the products through online reviews. An algorithm based on sentiment dictionaries is developed to identify the positive, neutral or negative sentiment orientation on the alternative product concerning the product feature in each review. According to the identified positive, neutral and negative sentiment orientations, an intuitionistic fuzzy number is constructed for representing the performance of an alternative product concerning a product feature. The ranking of alternative products is determined by intuitionistic fuzzy weighted averaging (IFWA) operator and preference ranking organization methods for enrichment evaluations II (PROMETHEE II). A case study is given to illustrate the use of the proposed method. The comparisons and experiments are further conducted to illustrate the characteristics and advantages of the proposed method. Converting the identified positive, neutral and negative sentiment orientations into intuitionistic fuzzy numbers is a new idea for processing and fusing a large number of sentiment orientations of online reviews. Based on the proposed method, decision support system can be developed to support the consumers’ purchase decisions more conveniently.
\end{abstract}

\footnotetext{
* Corresponding author at: Department of Information Management and Decision Sciences, School of Business Administration, Northeastern University, Shenyang 110167, China. Tel.:+86-24-8368 7753; Fax: +86-24-2389 1569.

E-mail address: zpfan@mail.neu.edu.cn (Z.P. Fan).
} 
Keywords: Product ranking; Online reviews; Sentiment analysis; Intuitionistic fuzzy number; IFWA operator

\section{Introduction}

With the development of e-commerce, more and more e-commerce websites and social media platforms encourage consumers to post online product reviews [1, 2]. For example, Amazon (http://www.amazon.cn/), Taobao (https://www.taobao.com/) and Automobile home (http://www.autohome.com.cn/) have provided channels for consumers to share their online product reviews. Some research results have shown that online product reviews have significant impacts on consumers' purchase decisions [3, 4]. That is, before making the purchase decisions, the consumers can visit the related websites and read online reviews on the alternative products. For example, a consumer wants to select a desirable sport utility vehicle (SUV) from the multiple alternative SUVs. The consumer may read the online reviews concerning the alternative SUVs so as to understand the alternative SUVs more clearly. But, it is difficult for the consumer to directly make the purchase decision based on online product reviews since the amount of online reviews is huge [5]. Therefore, to support the consumer's purchase decision, it is necessary to develop a method for ranking products through online reviews. Using the method, the sentiment orientation of each online review can be automatically identified, the performances of alternative products concerning each product feature can be analyzed, and the ranking of alternative products can be determined.

Till now, studies on ranking products through online reviews are still relatively scarce. Only limited literatures that directly or indirectly relate to this issue can be found [6-11]. Most of the existing methods for ranking products through online reviews consist of two parts, i.e., (1) analyzing online product reviews and (2) ranking products. The former is to determine the important product features or consumers' sentiment orientations by analyzing the online reviews, whereas the later is to obtain a ranking of products based on the analysis results of online reviews. These studies have made significant contributions to ranking products through online reviews. However, there are still some limitations when the existing methods are used. First, the purpose of the existing studies [6-11] is to rank similar products from the perspective of a third party. Thus, in most of the existing studies, the product features and the weights of product features are not considered or objectively determined based on the online reviews. But, when a consumer wants to buy a product from several alternative ones, the consumer may provide the product features and the weights of product features according to 
his/her own subjective preference. To support the consumer to select a desirable product, the ranking of alternative products should be determined according to the product features and the weights of product features provided by the consumer. Besides, in the existing studies [6-11], neutral sentiment orientations in the reviews are ignored. That will lead to the loss of valuable decision information. In fact, if a consumer posts a review with neutral sentiment orientation, then it means that the opinion of the consumer concerning the product is hesitant and uncertain. The information on hesitant or uncertain should not be ignored [12-14] since it is also valuable for the potential consumers to make a reasonable purchase decision. Intuitionistic fuzzy set theory proposed by Atanassov [15] is a useful tool to deal with vagueness, ambiguity and hesitation [16]. An intuitionistic fuzzy number can simultaneously reflect the degrees of support, hesitation and opposition of the evaluations or judgments about some specific events [16]. If the positive, neutral and negative sentiment orientations of the reviews on one product concerning one feature can be converted into an intuitionistic fuzzy number for representing the performance of the product concerning the feature, then the method for ranking products through online reviews can be developed based on the sentiment analysis technique and the intuitionistic fuzzy set theory. Thus, to support the consumers' purchase decisions, it is necessary to develop the algorithm for identifying the positive, neutral and negative sentiment orientations of the online reviews on products concerning different features, and propose the approach for ranking the alternative products based on intuitionistic fuzzy set theory.

The objective of this paper is to propose a method based on the sentiment analysis technique and the intuitionistic fuzzy set theory to rank products through online reviews. In this paper, we mainly focus on the online reviews in Chinese. This is because, Chinese is the second most used languages on the Internet currently, and appears to be the most used language on the web very soon [17]. Besides, this study is motivated by a practical case in a Chinese website, which will be shown in Section 5. The proposed method can be divided into two parts, i.e., the part of identifying sentiment orientations of the online reviews based on the sentiment analysis technique and the part of ranking the products based on the intuitionistic fuzzy set theory. In the former part, according to the alternative products and the product features concerned by the consumer, the online reviews of alternative products concerning the product features are crawled from the related website using the web crawler software; then, positive and negative sentiment dictionaries concerning each feature of the products are established according to the crawled online reviews and the HowNet sentiment dictionary (see: http://www.keenage.com/); further, according to the positive and negative sentiment 
dictionaries, the positive, neutral or negative sentiment orientation on the product concerning each feature in each review is identified by an algorithm. In the later part, by estimating important degree of posted time of each review, the weighted percentages of reviews with positive, neutral and negative sentiment orientations are calculated with respect to all the reviews on one product concerning one feature, and an intuitionistic fuzzy number can be formed according to the intuitionistic fuzzy set theory for representing the performance of the alternative product concerning the feature; then, according to the obtained intuitionistic fuzzy numbers and the weights of product features, an overall intuitionistic fuzzy number of each alternative product is calculated using intuitionistic fuzzy weighted averaging (IFWA) operator; the dominance degrees on pairwise comparisons of alternative products are obtained according to the overall intuitionistic fuzzy numbers, and a ranking of alternative products can be obtained using the preference ranking organization methods for enrichment evaluations II (PROMETHEE II).

The rest of this paper is arranged as follows. Section 2 provides an overview of the studies on sentiment analysis and the studies on ranking products through online reviews. Section 3 formulates the problem of ranking products through online reviews, and describes a resolution process for it. In Section 4, according to the resolution process, descriptions of the two parts of the proposed method are presented. In Section 5, a case study on ranking four automobile products is given to illustrate the use of the proposed method. Finally, Section 6 summarizes and highlights the major contributions of this paper.

\section{Related work}

To rank products through online reviews, it is necessary to identify the sentiment orientations of online reviews on alternative products concerning different features using sentiment analysis techniques. Then, based on the identified sentiment orientations, the studies on ranking products through online reviews can be conducted. Thus, the related work can be further classified into two aspects, i.e., (1) the studies on sentiment analysis, and (2) the studies on ranking products through online reviews. A brief literature review with respect to each aspect is given below.

\subsection{Studies on sentiment analysis}

Sentiment analysis and opinion mining are interrelated research areas, which are computational studies of opinions, sentiments, emotions, and attitude expressed in texts about 
an entity [18-21]. Sentiment analysis and opinion mining have many practical application backgrounds. By sentiment analysis and opinion mining of online texts, the government can acquire public opinions on public politics [22], companies can conduct market research and find weakness of product and service [23-25], and consumers can make better decisions on the purchasing of products or services [10, 11, 24]. In some existing studies, it is regarded that the expressions of sentiment analysis and opinion mining can be replaced by each other [18-21]. However, some scholars think there are slight difference between sentiment analysis and opinion mining $[18,26]$. It is regarded that opinion mining mainly focuses on extracting and analyzing people's opinion about an entity, while sentiment analysis mainly focuses on identifying the sentiment expressed in a text than analyzes it $[18,26]$. Here, we mainly focus on the sentiment analysis since the ranking of products is usually determined based on the identified sentiment orientations of online reviews.

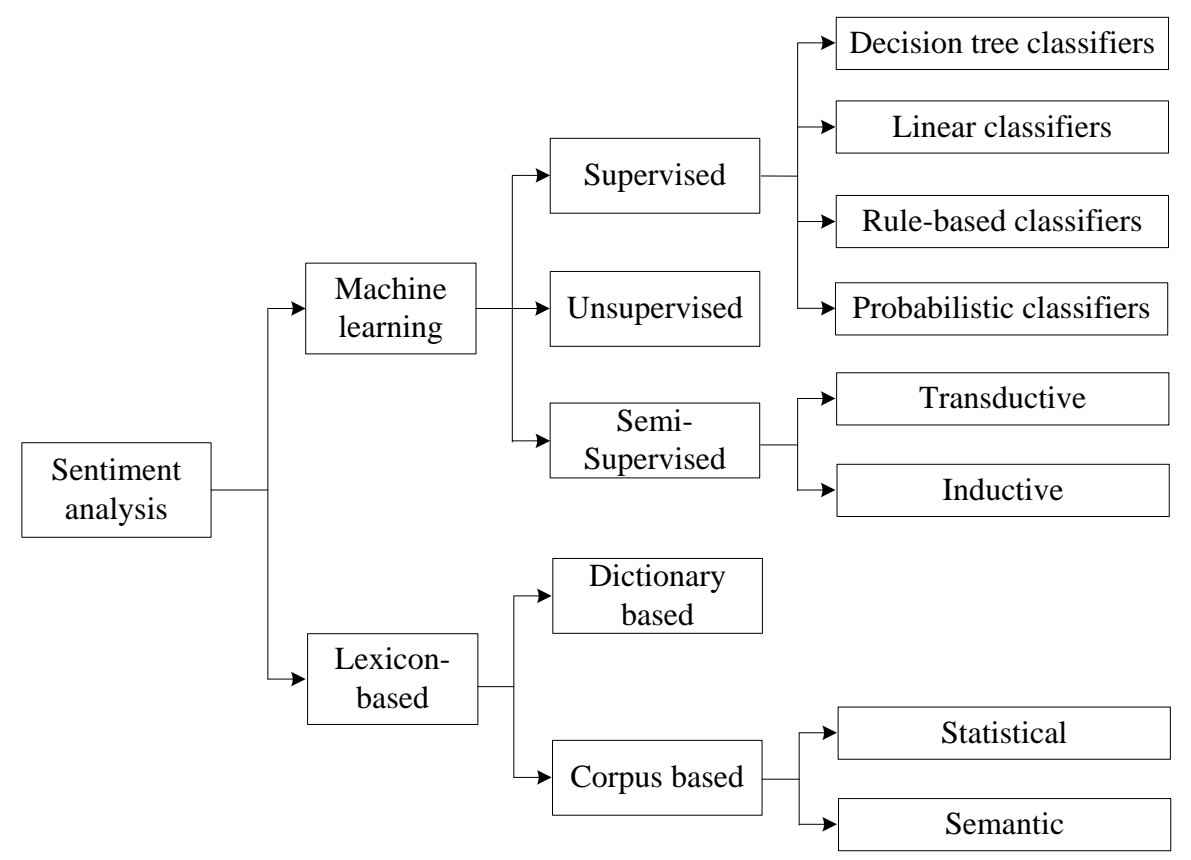

Fig. 1. The classification framework of the sentiment analysis techniques [18, 20, 27].

Sentiment analysis has attracted the attentions of some scholars. In the last few years, more than dozens of sentiment analysis techniques have been proposed [18-21]. According to the theoretical and technological foundations of the existing sentiment analysis techniques, the classification framework of the sentiment analysis techniques can be expressed by Fig. 1 [18, 20, 27]. It can be seen from Fig. 1 that the existing sentiment analysis techniques can be classified into two categories, i.e., sentiment analysis techniques based on machine learning [28-37] and lexicon-based sentiment analysis techniques [25, 38-41]. On the one hand, the sentiment analysis techniques based on machine learning can be further divided into three subclasses, i.e., (1) sentiment analysis techniques based on supervised machine learning 
[28-32], (2) sentiment analysis techniques based on unsupervised machine learning [33-35], and (3) sentiment analysis techniques based on semi-supervised machine learning [36, 37]. When the techniques based on supervised machine learning are used, a set of labeled training samples is needed [28-32]. By training different kinds of supervised machine learning algorithms using the samples, different sentiment classifiers can be obtained, such as decision tree classifiers, linear classifiers, rule-based classifiers and probabilistic classifiers [28-32]. For the situations that labeled training samples are absent, the techniques based on unsupervised machine learning can be used [33-35]. In the techniques based on unsupervised machine learning, the similarities of the texts are first measured according to keyword lists of categories, then the texts are clustered into multiple groups according to the similarities of the texts [33-35]. The techniques based on semi-supervised learning can be used for the situations with partially labeled data, such as partially sentences or partially features [36, 37]. On the other hand, the lexicon-based sentiment analysis techniques are further divided into two subclasses, i.e., (1) dictionary-based sentiment analysis techniques [38-40], and (2) corpus-based sentiment analysis techniques [25, 41]. The core of dictionary-based sentiment analysis techniques is to construct the sentiment dictionaries. In the dictionary-based sentiment analysis techniques, a small set of sentiment words is first determined manually. Then, by searching the synonyms and antonyms of the sentiment words in the well known corpora, such as the WordNet, HowNet or Thesaurus, new sentiment words are found and the number of the sentiment words is increased. The iterative process is carried out until no new sentiment words can be found, and the sentiment dictionaries are determined according to the obtained sentiment words [38-40]. The corpus-based sentiment analysis techniques are used to solve the problem of finding opinion words with context specific orientations. The core of the techniques is to find the syntactic patterns and a seed list of opinion words. For this, the statistical techniques are employed to find the co-occurrence patterns or seed opinion words, while the semantic techniques are used to determine the semantic values for different words according to the similarity of different words [25, 41]. To save the space of this paper, the details of these sentiment analysis techniques are not given. The detailed surveys of the existing studies can be found in literatures [18-21].

\subsection{Studies on ranking products through online reviews}

Although the sentiment analysis of online texts has attracted the attentions of scholars, studies on ranking products through online reviews are still relatively scarce. Only limited studies that directly or indirectly relate to this issue can be found [6-11]. 
Zhang et al. [6] proposed a method for ranking products through online reviews. In their method, a dynamic programming technique is proposed to identify subjective sentences and comparative sentences in online product reviews, where a subjective sentence represents the subjective opinion of a consumer on a product, and a comparative sentence represents a comparison relationship on a pair of products. Then, the sentiment analysis technique is used to identify the positive or negative sentiment orientation of each subjective sentence and each comparative sentence. Further, according to the obtained sentiment orientations, the numbers of different kinds of sentences (positive subjective sentences, negative subjective sentences, positive comparative sentences and negative comparative sentences) concerning multiple products are obtained, and a directed and weighted product graph is constructed which simultaneously reflects the subjective opinions and comparison relationships on the products. Finally, according to the directed and weighted product graph, a ranking of products is determined using an improved page rank algorithm. On the basis of literature [6], Zhang et al. [7] further proposed a feature-based method for ranking products through online reviews. In the method, it is assumed that multiple important features of the kind of products have been first identified manually or automatically, and then the sentences concerning each feature are extracted from the online reviews. Further, with respect to the sentences concerning each feature, a procedure similar to the one reported in literature [6] is used to determine a ranking of products. Finally, the obtained multiple rankings of products with respect to different features are simultaneously provided to the consumers to support their purchase decisions. Thereafter, Zhang et al. [8] further improved the methods proposed in literatures [6] and [7] by considering the important degrees or weights of different reviews. In the improved method, the weight of each review is determined according to the helpfulness and the age importance of the review, where the helpfulness is estimated according to the number of helpful votes received by the review, and the age importance is measured according to the posted date of the review. Peng et al. [9] proposed a fuzzy PROMETHEE approach for ranking products through online reviews in Chinese. In the approach, first, the synonyms concerning each product feature are determined by calculating similarity degrees of different Chinese words and defining the threshold of similarity degrees. Then, the set of important product features is determined according to the total frequency of the synonyms concerning each feature in all the reviews. Further, a fuzzy decision matrix on multiple products concerning the important product features is obtained according to the subjective evaluations of several domain experts. Finally, according to the fuzzy decision matrix, a ranking of products is determined by fuzzy PROMETHEE method. Chen et al. [10] proposed a market structure visualization approach 
based on online product reviews. In their approach, the online product reviews are first classified into positive reviews and negative reviews according to the polarity, and the further analysis is conducted based on the positive reviews and the negative reviews, respectively. On the one hand, based on the positive reviews, topic modeling and scree plot technique are used to determine the topic distribution matrix with filtered noises. Then, according to the topic distribution matrix, the weight matrix of all brands and the weights of important topics are determined. Further, according to the obtained weight matrix of all brands and the weights of important topics, a perceptual map of market structure is built by the multi-dimensional scaling method. Moreover, a ranking of products can be obtained using technique for order preference by similarity to an ideal solution (TOPSIS) approach. On the other hand, based on the negative reviews, another perceptual map and another ranking of products can be obtained by the similar procedure of the positive reviews. Najmi et al. [11] proposed a comprehensive method for ranking products through online reviews. In the method, the ranking score of each product is determined by combining the brand score and the review score, where the brand score is calculated by an improved page rank algorithm and the review score is calculated according to the results of sentiment analysis and usefulness analysis of each online review.

The existing studies have made significant contributions to sentiment analysis and ranking products through online reviews. However, in most of the existing studies on sentiment analysis, only two kinds of sentiment orientations are identified, i.e., the sentiment orientation of each text or sentence is identified as either positive or negative. Besides, in the existing studies on ranking products through online reviews, neutral sentiment orientations in the online reviews are also ignored. That will lead to the loss of valuable decision information. Thus, to support the consumers' purchase decisions, it is necessary to develop the method that can identify the positive, neutral and negative sentiment orientations of online reviews, and can rank alternative products according to the identified sentiment orientations and the weights of product features provided by the consumer.

\section{The problem of ranking products through online reviews}

In this section, we first formulate the problem of ranking products through online reviews, and then briefly describe a resolution process for it.

\subsection{Description of the problem of ranking products through online reviews}

The problem of ranking products through online reviews is vividly shown in Fig. 2. 
Consider a consumer wants to buy a product, such as a SUV. By a preliminary investigation, several acceptable products are determined, which are regarded as the alternative products. But, the consumer wavers among the several alternative products since the limited knowledge and expertise. To select a desirable product, several product features are considered, which are determined according to the consumer's personal preference. Further, the weights of these features can be directly determined by the assignment of the consumer or indirectly obtained using existing procedures such as analytic hierarchy process (AHP) [42]. To support the consumer's purchase decision, a large number of online reviews of the alternative products concerning the product features are crawled from the related website. The problem concerned in this paper is how to rank the alternative products based on the online reviews and the feature weights provided by the consumer.

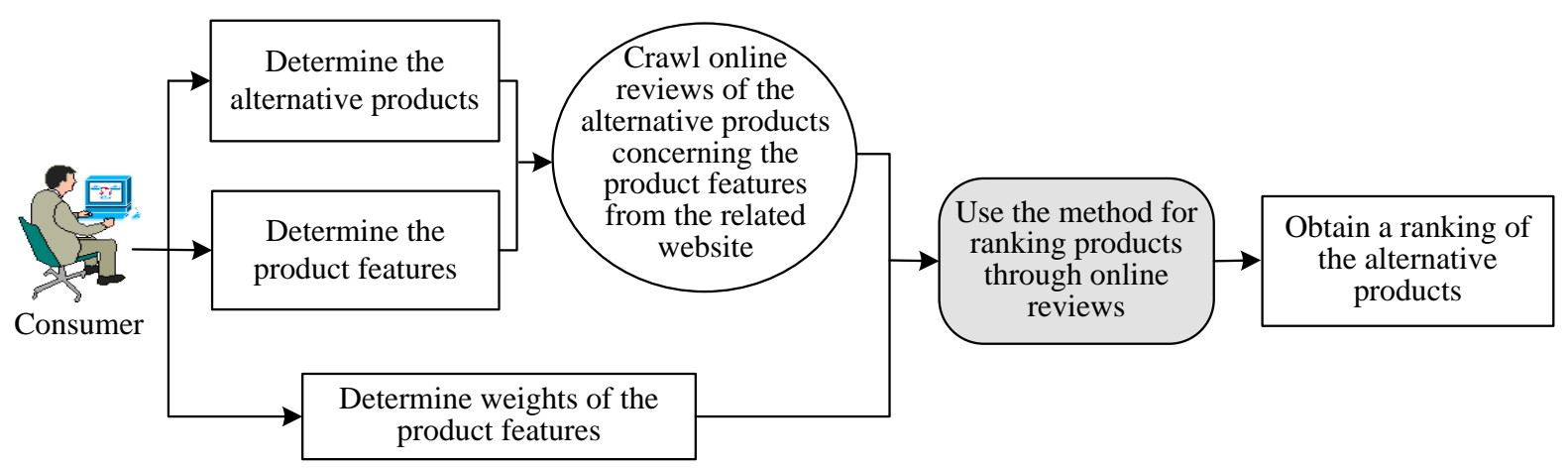

Fig. 2. The problem of ranking products through online reviews.

The following notations are used to denote the sets and variables in the problem, which will be used throughout this paper.

- $A=\left\{A_{1}, A_{2}, \cdots, A_{n}\right\}$ : the set of $n$ alternative products, where $A_{i}$ denotes the ith alternative product, $i=1,2, \ldots, n$. Usually, the set $A$ can be determined by the consumer.

- $F=\left\{f_{1}, f_{2}, \cdots, f_{m}\right\}$ : the set of $m$ product features, where $f_{j}$ denotes the $j$ th product feature, $j=1,2, \ldots, m$. Usually, the set $F$ can be determined by the consumer according to his/her personal preference.

- $w=\left(w_{1}, w_{2}, \cdots, w_{m}\right)$ : the vector of feature weights, where $w_{j}$ denotes the weight of feature $f_{j}$, such that $w_{j} \geqslant 0$ and $\sum_{j=1}^{m} w_{j}=1, j=1,2, \ldots, m$. The vector of feature weights can be directly determined by the assignment of the consumer or indirectly obtained using existing procedures such as AHP [42].

- $Q=\left(q_{1}, q_{2}, \ldots, q_{n}\right)$ : the vector of numbers of the online reviews concerning alternative 
products, where $q_{i}$ denotes the number of the online reviews concerning alternative product $A_{i}, \quad i=1,2, \ldots, n$.

- $D_{i k}=\left(D_{i k}^{1}, D_{i k}^{2}, \ldots, D_{i k}^{m}\right)$ : the $k$ th online review concerning alternative product $A_{i}$, where $D_{i k}^{j}$ denotes the sentence concerning feature $f_{j}$ in the $k$ th online review of alternative product $A_{i}, i=1,2, \ldots, n, j=1,2, \ldots, m, k=1,2, \ldots, q_{i}$.

The problem concerned in this paper is how to rank alternative products $A_{1}, A_{2}, \cdots, A_{n}$ based on the online review $D_{i k}$ and feature weight $w_{j}, i=1,2, \ldots, n, j=1,2, \ldots, m$, $k=1,2, \ldots, q_{i}$.

\subsection{Resolution process for the problem of ranking products through online reviews}

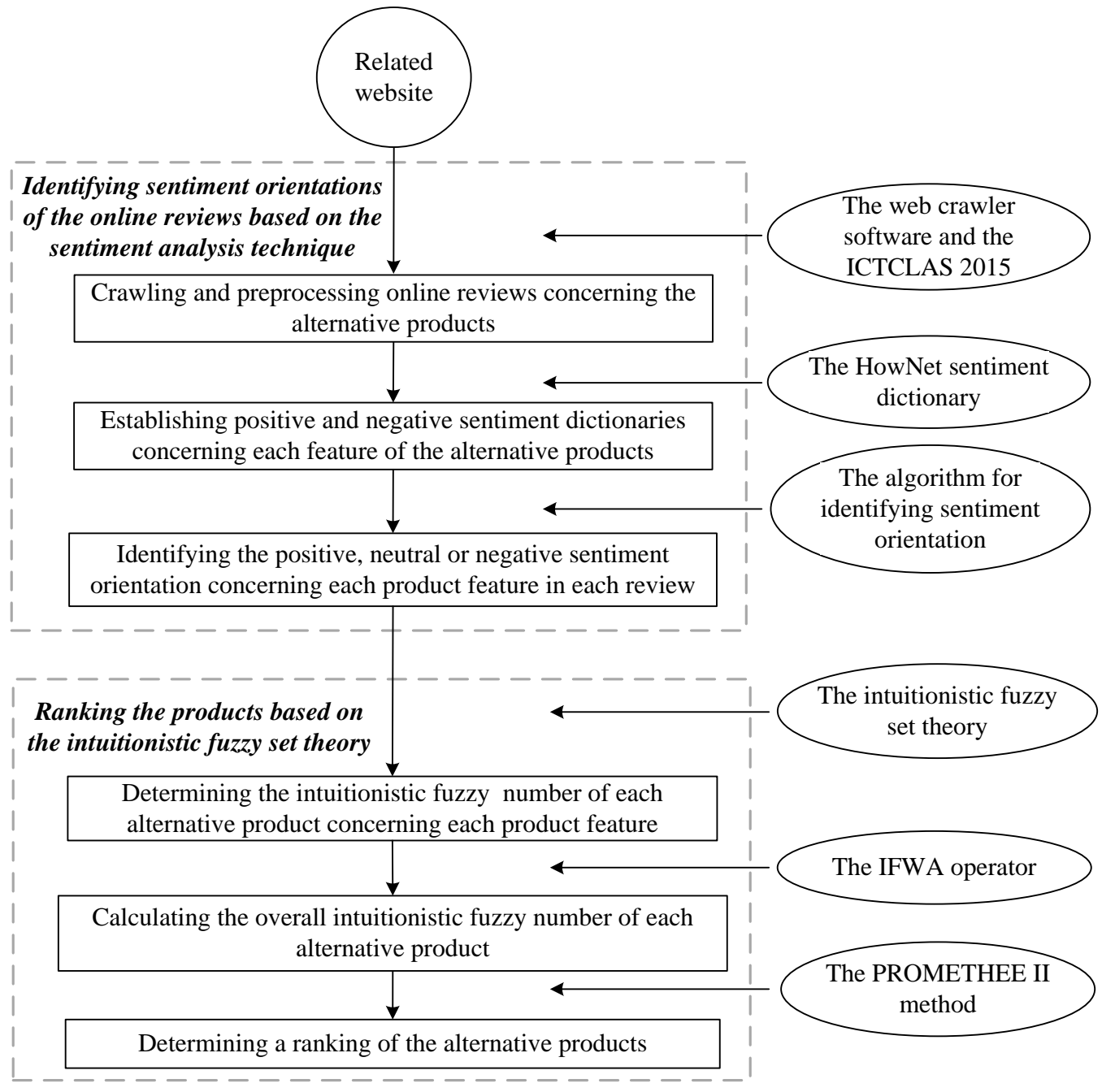

Fig. 3. The resolution process for the problem of ranking products through online reviews.

To solve the above problem, a resolution process is proposed as shown in Fig. 3. It can be seen from Fig. 3 that the resolution process can be divided into two parts, i.e., (1) identifying 
sentiment orientations of the online reviews based on the sentiment analysis technique, and (2) ranking the products based on the intuitionistic fuzzy set theory. In the former part, according to the alternative products and the product features concerned by the consumer, the online reviews of the alternative products concerning the product features are crawled from the related website using the web crawler software and are preprocessed using the ICTCLAS 2015 (Institute of Computing Technology, Chinese Lexical Analysis System, see: http://ictclas.nlpir.org/); then, positive and negative sentiment dictionaries concerning each feature of the alternative products are established according to the obtained online reviews and the HowNet sentiment dictionary; further, the positive, neutral or negative sentiment orientation on the product concerning each product feature in each review is identified by the algorithm for identifying sentiment orientation. In the later part, by estimating important degree of posted time of each review, the weighted percentages of reviews with positive, neutral and negative sentiment orientations are calculated with respect to all the reviews on one product concerning one feature, and an intuitionistic fuzzy number can be determined according to the intuitionistic fuzzy set theory for representing the performance of the alternative product concerning the feature; then, an overall intuitionistic fuzzy number of each alternative product is calculated by aggregating the intuitionistic fuzzy numbers of the product concerning multiple product features and the weights of the multiple product features using the IFWA operator; further, according to the obtained overall intuitionistic fuzzy numbers, the dominance degrees on pairwise comparisons of the alternative products are obtained and a ranking of the alternative products can be determined using the PROMETHEE II method.

\section{The method for ranking products through online reviews}

According to the resolution process shown in Fig. 3, a description of the proposed method for ranking products through online reviews will be given in this section, where the description of the part of identifying sentiment orientations of the online reviews based on the sentiment analysis technique will be given in Section 4.1, and the description of the part of ranking products based on the intuitionistic fuzzy set theory will be given in Section 4.2.

\subsection{Identifying sentiment orientations of the online reviews based on the sentiment analysis technique}

According to the literature review in Section 2.1, we know that the existing sentiment analysis techniques can be classified into two categories, i.e., sentiment analysis techniques 
based on machine learning [28-37] and lexicon-based sentiment analysis techniques [25, 38-41]. The results of existing studies have shown that the sentiment analysis techniques based on machine learning are more suitable to be used in document-level sentiment analysis, while the lexicon-based sentiment analysis techniques are more suitable to be used in sentence-level sentiment analysis. Usually, in an online product review, only one or two sentences are used to express the opinion on the product concerning a feature, which belong to the sentence-level. Thus, in this paper, the idea of dictionary-based sentiment analysis techniques is used to identify the positive, neutral and negative sentiment orientations on the products concerning the product features in online reviews. For this, the part can be further divided into three segments: (1) crawling and preprocessing online reviews concerning the alternative products; (2) establishing positive and negative sentiment dictionaries concerning each feature of the alternative products; and (3) identifying the positive, neutral or negative sentiment orientation concerning each product feature in each review. Detail description of each segment is given below.

\subsubsection{Crawling and preprocessing online reviews concerning the alternative products}

In this paper, the crawler software is used to obtain online reviews and the ICTCLAS 2015 is used to preprocess the obtained online reviews. The details are described as follows.

\section{(1) Crawling online reviews concerning the alternative products}

According to the set of alternative products, i.e., $A=\left\{A_{1}, A_{2}, \cdots, A_{n}\right\}$, the online product reviews can be crawled from the related website. Till now, some crawlers have been developed, which can be used to obtain the reviews. Detailed introduction on crawlers can be found on Wikipedia (https://en.wikipedia.org/wiki/Web_crawler). Currently, some websites encourage the consumers to post their reviews according to a pre-established framework of product features. For example, Automobile home constructs a framework on the features of automobiles, and encourages the consumers to post their reviews according to the framework. Fig. 4 shows an example of the product review crawled from Automobile home, where the review is posted according to the pre-established framework on automobile product features. Besides, for the situations that reviews are not posted according to the pre-established framework of features, some existing techniques can be used to extract sentences from each

review according to the set of product features $F=\left\{f_{1}, f_{2}, \cdots, f_{m}\right\}[17,43]$. Thus, in this study, we suppose that the sentences concerning the product features have been extracted from the online reviews. Therefore, the $k$ th online review of alternative product $A_{i}$ can be expressed by $D_{i k}=\left(D_{i k}^{1}, D_{i k}^{2}, \ldots, D_{i k}^{m}\right)$, where $D_{i k}^{j}$ denotes the sentence concerning feature 
$f_{j}$ in review $D_{i k}, i=1,2, \ldots, n, j=1,2, \ldots, m, k=1,2, \ldots, q_{i}$. If there is no sentence concerning product feature $f_{j}$ in the review $D_{i k}$, then we denote $D_{i k}^{j}=\varnothing, i=1,2, \ldots, n$, $j=1,2, \ldots, m, k=1,2, \ldots, q_{i}$.

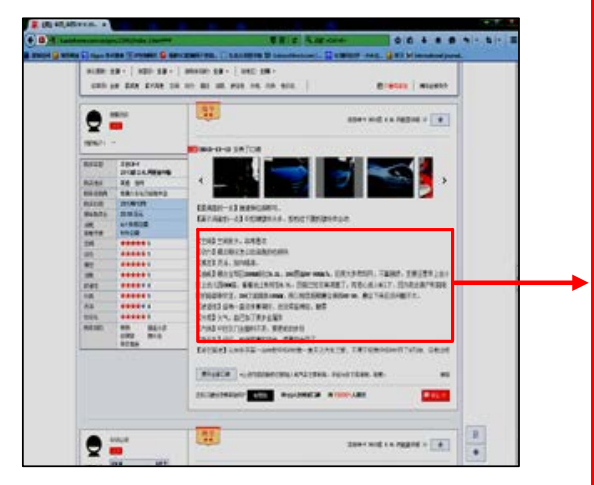

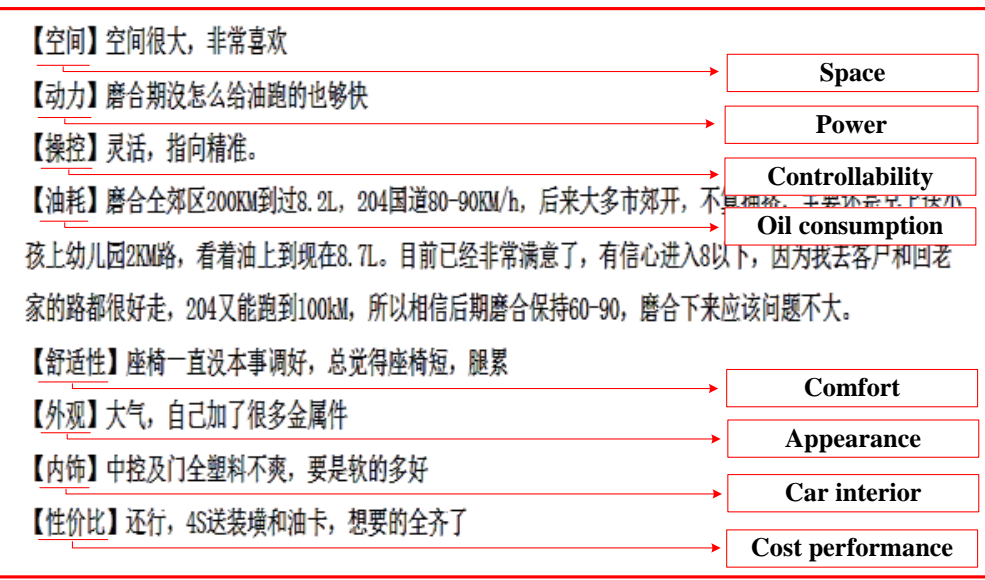

Fig. 4. An example of the product review crawled from Automobile home

(http://k.autohome.com.cn/spec/15963/index_3.html\#\#\#).

(2) Preprocessing the online reviews

To identify the sentiment orientations of the online reviews, it is necessary to preprocess the obtained online reviews. The preprocessing including the two processes, namely: (i) word segmentation and part-of-speech (POS) tagging, and (ii) stop words removing. The details are given below.

(i) Word segmentation and POS tagging. The online reviews concerning the alternative products are imported into the ICTCLAS 2015 for word segmentation and POS tagging. Through word segmentation and POS tagging, each sentence in the online reviews is decomposed into several words, and the POS of each word is tagged after the word. For example, if Chinese sentence “油耗不算大” (i.e., “fuel-consumption is not big”) is imported into the ICTCLAS 2015, then the output result is “油耗/n 不/d 算/v 大/a” (i.e., “fuel-consumption /n, is /v, not /d, big /a”), where “/n” denotes “noun”; “/d” denotes "adverb”; “/v" denotes "verb”; and “/a” denotes "adjective”.

(ii) Stop words removing. Stop words refer to the words that frequently appear in the text but do not carry significant information. To improve the efficiency and effectiveness of sentiment analysis, stop words need to be removed beforehand. In this paper, the words obtained from word segmentation and POS tagging are compared with stop word list (see: http://www.datatang.com/data/19300). If a word belongs to the stop word list, then the word is deleted. After removing stop words, the notional words of each online review can be 
determined, noted as $\bar{W}_{i k}=\left(\bar{W}_{i k}^{1}, \bar{W}_{i k}^{2}, \ldots, \bar{W}_{i k}^{m}\right)$, where $\bar{W}_{i k}^{j}$ denotes the set of notional words in sentence $D_{i k}^{j}, i=1,2, \ldots, n, j=1,2, \ldots, m, k=1,2, \ldots, q_{i}$. Specially, if $D_{i k}^{j}=\varnothing$, then we denote $\bar{W}_{i k}^{j}=\varnothing, \quad i=1,2, \ldots, n, \quad j=1,2, \ldots, m, k=1,2, \ldots, q_{i}$.

\subsubsection{Establishing positive and negative sentiment dictionaries concerning each feature of the} alternative products

Usually, the positive or negative sentiment dictionaries concerning different features may be different. In fact, a word can simultaneously belong to the positive sentiment dictionary of a feature and the negative sentiment dictionary of another feature. For example, “高” (high) belongs to the positive sentiment dictionary of feature "cost performance" and the negative sentiment dictionary of feature “oil consumption”. Therefore, it is necessary to establish the positive and negative sentiment dictionaries for each feature, respectively.

Let $\bar{W}_{i}^{j}$ denote the set of notional words concerning feature $f_{j}$ in the reviews on alternative product $A_{i}$, and $\bar{W}^{j}$ denote the set of notional words concerning product feature $f_{j}$ in all the reviews. Then, $\bar{W}_{i}^{j}$ and $\bar{W}^{j}$ can be respectively represented by

$$
\begin{aligned}
\bar{W}_{i}^{j} & =\bar{W}_{i 1}^{j} \cup \bar{W}_{i 2}^{j} \cup \cdots \cup \bar{W}_{i q_{i}}^{j}, \quad i=1,2, \ldots, n, \quad j=1,2, \ldots, m, \\
\bar{W}^{j} & =\bar{W}_{1}^{j} \cup \bar{W}_{2}^{j} \cup \cdots \cup \bar{W}_{n}^{j}, \quad j=1,2, \ldots, m .
\end{aligned}
$$

Further, let $\bar{W}^{\prime j}=\left\{W_{1}^{j}, W_{2}^{j}, \cdots, W_{h_{j}}^{j}\right\}$ denote the set of sentiment words concerning feature $f_{j}$, where $h_{j}$ denotes the number of sentiment words concerning feature $f_{j}$ in all the reviews, $j=1,2, \ldots, m$. According to the existing studies $[44,45], \bar{W}^{\prime j}$ can be obtained by extracting the adjectives, adverbs and verbs from the set of notional words $\bar{W}^{j}$, $j=1,2, \ldots, m$. Moreover, let $\bar{W}_{\mathrm{HowNet}}^{+}=\left\{W_{1}^{\mathrm{HN}+}, W_{2}^{\mathrm{HN}+}, \cdots, W_{4566}^{\mathrm{HN}+}\right\}$ and $\bar{W}_{\mathrm{HowNet}}^{-}=\left\{W_{1}^{\mathrm{HN}-}, W_{2}^{\mathrm{HN}-}\right.$, $\left.\cdots, W_{4370}^{\mathrm{HN}-}\right\}$ respectively denote the sets of positive and negative sentiment words in the HowNet sentiment dictionary (see: http://www.keenage.com/), where $\bar{W}_{\text {Hownet }}^{+}$and $\bar{W}_{\text {HowNet }}^{-}$ include 4566 positive sentiment words and 4370 negative sentiment words, respectively. Let $\bar{W}_{j}^{+}$and $\bar{W}_{j}^{-}$denote the positive sentiment dictionary and the negative sentiment dictionary concerning feature $f_{j}$, respectively. Then, $\bar{W}_{j}^{+}$and $\bar{W}_{j}^{-}$can be established according to $\bar{W}_{\text {HowNet }}^{+}, \bar{W}_{\text {HowNet }}^{-}$and $\bar{W}^{\prime j}$, i.e.,

$$
\bar{W}_{j}^{+}=\bar{W}_{\text {HowNet }}^{+} \cap \bar{W}^{\prime j}, \quad j=1,2, \ldots, m,
$$




$$
\bar{W}_{j}^{-}=\bar{W}_{\text {HowNet }}^{-} \cap \bar{W}^{\prime j}, \quad j=1,2, \ldots, m .
$$

For the situations that the sentiment orientations of some sentiment words can not be correctly identified using the HowNet sentiment dictionary, the manual identification can be employed to determine the positive or negative sentiment orientation of the sentiment words concerning different features.

\subsubsection{Identifying the positive, neutral or negative sentiment orientation concerning each} product feature in each review

In this section, an algorithm is proposed to identify the positive, neutral or negative sentiment orientation on the product concerning each product feature in each review. The main idea of the algorithm is: the sentiment orientation of a sentence depends on the sentiment words in the sentence, if there are only positive sentiment words in the sentence, then the sentiment orientation of the sentence is regarded as positive; if there are only negative sentiment words in the sentence, then the sentiment orientation of the sentence is regarded as negative; if there are no sentiment words or there are both positive and negative sentiment words in the sentence, then the sentiment orientation of the sentence is regarded as neutral; if there are negation words in the sentence, then the sentiment orientation of the sentence will be reversed. Detailed description of the algorithm is given below.

Let $\bar{W}_{\text {neg }}$ denotes the set of negation words (see: http://www.datatang.com/data/44198). Let $S_{i k}^{j}=\left(\alpha_{i k}^{j}, \beta_{i k}^{j}, \gamma_{i k}^{j}\right)$ denote the indicator vector of sentiment orientation of sentence $D_{i k}^{j}$, where $\alpha_{i k}^{j}, \beta_{i k}^{j}$ and $\gamma_{i k}^{j}$ are indicator variables for positive, neutral and negative sentiment orientations, respectively, $\alpha_{i k}^{j}, \beta_{i k}^{j}, \gamma_{i k}^{j} \in\{0,1\}, \quad \alpha_{i k}^{j}+\beta_{i k}^{j}+\gamma_{i k}^{j}=0$ or $1, \quad i=1,2, \ldots, n$, $j=1,2, \ldots, m, k=1,2, \ldots, q_{i}$. The values of $S_{i k}^{j}=\left(\alpha_{i k}^{j}, \beta_{i k}^{j}, \gamma_{i k}^{j}\right)$ have four cases, i.e., $S_{i k}^{j}=(1,0,0), \quad S_{i k}^{j}=(0,1,0), S_{i k}^{j}=(0,0,1) \quad$ and $\quad S_{i k}^{j}=(0,0,0)$, where $S_{i k}^{j}=(1,0,0)$, $S_{i k}^{j}=(0,1,0)$ and $S_{i k}^{j}=(0,0,1)$ respectively denote that sentence $D_{i k}^{j}$ expresses the positive, neutral and negative sentiment orientation; specially, $S_{i k}^{j}=(0,0,0)$ denotes $D_{i k}^{j}=\varnothing$. The pseudo code of the algorithm for identifying the sentiment orientation of $D_{i k}^{j}$ is shown in Algorithm 1, where $S_{i k}^{j+}, S_{i k}^{j-}$ and $S_{i k}^{\text {jneg }}$ are intermediate variables.

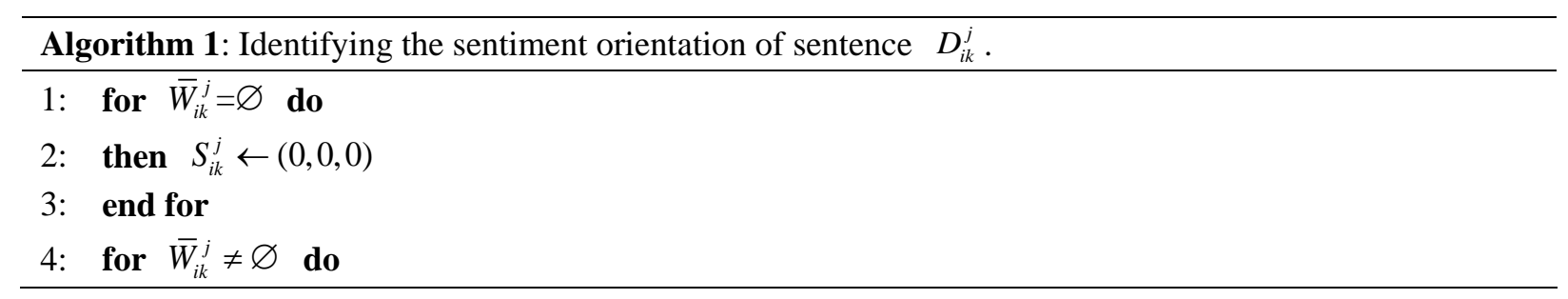




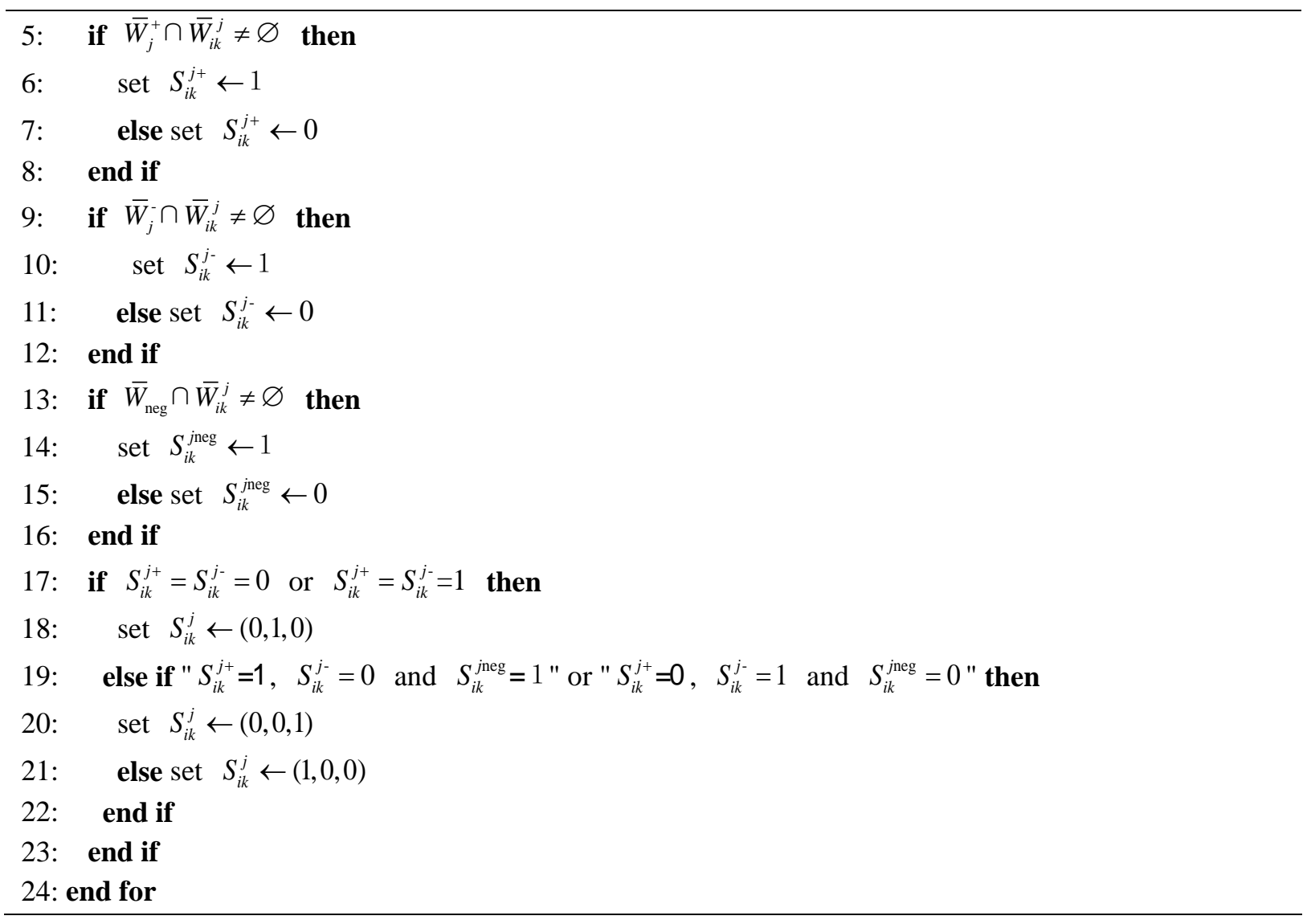

\subsection{Ranking the products based on the intuitionistic fuzzy set theory}

In this section, an approach to ranking the alternative products based on the intuitionistic fuzzy set theory is proposed. The approach includes the three segments: (1) determining the intuitionistic fuzzy number of each alternative product concerning each product feature; (2) calculating the overall intuitionistic fuzzy number of each alternative product; and (3) determining a ranking of the alternative products. Detail description of each segment is given below.

\subsubsection{Determining the intuitionistic fuzzy number of each alternative product concerning} each product feature

Intuitionistic fuzzy set theory [15] is a useful tool to deal with vagueness, ambiguity and hesitation [16]. An intuitionistic fuzzy number can simultaneously reflect the degrees of support, hesitation and opposition of the evaluations or judgments about some specific events [16]. If a review with positive sentiment orientation is regard as a vote in support, a review with neutral sentiment orientation is regard as hesitation, and a review with negative sentiment orientation is regard as a vote in opposition, then an intuitionistic fuzzy number can be constructed for representing the performance of an alternative product concerning a product feature. Based on intuitionistic fuzzy set theory [15], a large number of sentiment 
orientations of online reviews on an alternative product concerning a product feature can be simply and completely represented by an intuitionistic fuzzy number. Besides, since the technical update and improvement, there could be slight differences between the products sold at present and those sold before. Thus, compared with the sentiment orientations in the earlier reviews, the sentiment orientations in the newer reviews are more consistent with those for the products sold at present. For this, it is necessary to distinguish the posted time of the online reviews, i.e., the greater important degree of the posted time should be assigned to the newer online review. Let $w_{i k}^{j}$ denote the important degree of the posted time of review $D_{i k}^{j}$, $i=1,2, \ldots, n, j=1,2, \ldots, m, k=1,2, \ldots, q_{i}$. According to the study of Najmi et al. [11], $w_{i k}^{j}$ can be calculated by

$$
w_{i k}^{j}=e^{\left(T_{i k}^{j}-T_{i}\right) /\left(T_{C}-T_{i}\right)}, \quad i=1,2, \ldots, n, \quad j=1,2, \ldots, m, k=1,2, \ldots, q_{i},
$$

where $T_{i k}^{j}$ denotes the posted time of review $D_{i k}^{j}, T_{i}$ denotes the release time of product $A_{i}, T_{C}$ denote the current time, $i=1,2, \ldots, n, j=1,2, \ldots, m, k=1,2, \ldots, q_{i}$.

Let $q_{i j}^{\text {pos }}, q_{i j}^{\text {neu }}$ and $q_{i j}^{\text {neg }}$ denote the weighted frequencies of reviews with positive, neutral and negative sentiment orientations on alternative product $A_{i}$ concerning feature $f_{j}$, respectively. The values of $q_{i j}^{\text {pos }}, q_{i j}^{\text {neu }}$ and $q_{i j}^{\text {neg }}$ can be respectively calculated by

$$
\begin{aligned}
& q_{i j}^{\text {pos }}=\sum_{k=1}^{q_{i}} w_{i k}^{j} \alpha_{i k}^{j}, \quad i=1,2, \ldots, n, \quad j=1,2, \ldots, m, \\
& q_{i j}^{\text {neu }}=\sum_{k=1}^{q_{i}} w_{i k}^{j} \beta_{i k}^{j}, \quad i=1,2, \ldots, n, \quad j=1,2, \ldots, m, \\
& q_{i j}^{\text {neg }}=\sum_{k=1}^{q_{i}} w_{i k}^{j} \gamma_{i k}^{j}, \quad i=1,2, \ldots, n, \quad j=1,2, \ldots, m .
\end{aligned}
$$

Let $p_{i j}^{\text {pos }}, p_{i j}^{\text {neu }}$ and $p_{i j}^{\text {neg }}$ denote the weighted percentages of reviews with positive, neutral and negative sentiment orientations in all the reviews on alternative product $A_{i}$ concerning feature $f_{j}$, respectively. Then, $p_{i j}^{\text {pos }}, p_{i j}^{\text {neu }}$ and $p_{i j}^{\text {neg }}$ can be respectively calculated by Eqs. (9)-(11), i.e.,

$$
\begin{aligned}
& p_{i j}^{\text {pos }}=\frac{q_{i j}^{\text {pos }}}{q_{i j}^{\text {pos }}+q_{i j}^{\text {neu }}+q_{i j}^{\text {neg }}}, \quad i=1,2, \ldots, n, \quad j=1,2, \ldots, m, \\
& p_{i j}^{\text {neu }}=\frac{q_{i j}^{\text {neu }}}{q_{i j}^{\text {pos }}+q_{i j}^{\text {neu }}+q_{i j}^{\text {neg }}}, \quad i=1,2, \ldots, n, \quad j=1,2, \ldots, m,
\end{aligned}
$$




$$
p_{i j}^{\text {neg }}=\frac{q_{i j}^{\text {neg }}}{q_{i j}^{\text {pos }}+q_{i j}^{\text {neu }}+q_{i j}^{\text {neg }}}, \quad i=1,2, \ldots, n, \quad j=1,2, \ldots, m .
$$

Obviously, we know $p_{i j}^{\text {pos }}+p_{i j}^{\text {neu }}+p_{i j}^{\text {neg }}=1 \quad$ and $\quad p_{i j}^{\text {pos }}, p_{i j}^{\text {neu }}, p_{i j}^{\text {neg }} \geq 0, \quad i=1,2, \ldots, n$, $j=1,2, \ldots, m$.

In this paper, the reviews with positive sentiment orientation are regard as votes in support, and reviews with negative sentiment orientation are regard as votes in opposition. Thus, the $p_{i j}^{\text {pos }}$ and $p_{i j}^{\text {neg }}$ can be regarded as the support and opposition degrees of alternative product $A_{i}$ concerning feature $f_{j}, i=1,2, \ldots, n, j=1,2, \ldots, m$. Therefore, according to the physical interpretation of intuitionistic fuzzy number [12, 15], an intuitionistic fuzzy number $X_{i j}=\left(\mu_{i j}, v_{i j}\right)$ can be constructed for representing the performance of alternative product $A_{i}$ concerning feature $f_{j}$, where $\mu_{i j}=p_{i j}^{\text {pos }}$ and $v_{i j}=p_{i j}^{\text {neg }}$ respectively denote the support and opposition degrees of alternative product $A_{i}$ concerning feature $f_{j}, i=1,2, \ldots, n$, $j=1,2, \ldots, m$.

\subsubsection{Calculating the overall intuitionistic fuzzy number of each alternative product}

According to intuitionistic fuzzy numbers $x_{i 1}=\left(\mu_{i 1}, v_{i 1}\right), x_{i 2}=\left(\mu_{i 2}, v_{i 2}\right), \cdots$, $X_{i m}=\left(\mu_{i m}, v_{i m}\right)$ and feature weights $w_{1}, w_{2}, \cdots, w_{m}$ provided by the consumer, an overall intuitionistic fuzzy number of alternative product $A_{i}$ can be calculated. Several operators have been proposed for aggregating the multiple intuitionistic fuzzy numbers, such as IFWA operator and intuitionistic fuzzy ordered weighted averaging (IFOWA) operator, etc [12, 46]. Different aggregation operators are based on different assumptions and are suitable to be used in different kinds of problems. For example, the IFWA operator is suitable to be used in the problems that the weights are assigned to the product features, whereas the IFOWA operator is suitable to be used in the problems that the weights are assigned to the ranking positions of feature values $[12,46]$. In this paper, the weights are assigned to the product features, thus the IFWA operator $[12,46]$ is used to calculate an overall intuitionistic fuzzy number of each alternative product, i.e.,

$$
z_{i}=w_{1} x_{i 1} \oplus w_{2} x_{i 2} \oplus \cdots \oplus w_{m} x_{i m}=\left(1-\prod_{j=1}^{m}\left(1-\mu_{i j}\right)^{w_{j}}, \prod_{j=1}^{m} v_{i j}^{w_{j}}\right), \quad i=1,2, \ldots, n,
$$

where $1-\prod_{j=1}^{m}\left(1-\mu_{i j}\right)^{w_{j}}$ and $\prod_{j=1}^{m} v_{i j}^{w_{j}}$ represent the overall support degree and the overall 
opposition degree of product $A_{i}$, respectively.

\subsubsection{Determining a ranking of the alternative products}

By Eq. (12), the overall intuitionistic fuzzy numbers of alternative products $A_{i}$ and $A_{i^{\prime}}$ can be respectively represented by $z_{i}=\left(1-\prod_{j=1}^{m}\left(1-\mu_{i j}\right)^{w_{j}}, \prod_{j=1}^{m} v_{i j}^{w_{j}}\right) \quad$ and $Z_{i^{\prime}}=\left(1-\prod_{j=1}^{m}\left(1-\mu_{i^{\prime}}\right)^{w_{j}}, \prod_{j=1}^{m} v_{i^{\prime} j}^{w_{j}}\right), \quad i, i^{\prime}=1,2, \ldots, n, \quad i \neq i^{\prime}$. Let $p_{i i^{\prime}}$ denote the dominance degree of $Z_{i}$ over $Z_{i^{\prime}}$, which can be regarded as the dominance degree of product $A_{i}$ over product $A_{i^{\prime}}$. Without loss of generality, we suppose $1-\prod_{j=1}^{m}\left(1-\mu_{i j}\right)^{w_{j}} \geqslant 1-\prod_{j=1}^{m}\left(1-\mu_{i^{\prime} j}\right)^{w_{j}}$. Then, according to the study of $\mathrm{Yu}$ and $\mathrm{Xu}[46], p_{i i^{\prime}}$ can be calculated by the following Eqs. (13) and (14), i.e.,

(i) If $1-\prod_{j=1}^{m}\left(1-\mu_{i j}\right)^{w_{j}}>1-\prod_{j=1}^{m}\left(1-\mu_{i^{\prime} j}\right)^{w_{j}}$, then

$$
p_{i i^{\prime}}=\frac{\prod_{j=1}^{m}\left(1-\mu_{i^{\prime} j}\right)^{w_{j}}-\prod_{j=1}^{m}\left(1-\mu_{i j}\right)^{w_{j}}}{\prod_{j=1}^{m}\left(1-\mu_{i^{\prime} j}\right)^{w_{j}}-\prod_{j=1}^{m}\left(1-\mu_{i j}\right)^{w_{j}}+\max \left(\prod_{j=1}^{m} v_{i j}^{w_{j}}-\prod_{j=1}^{m} v_{i^{\prime} j}^{w_{j}}, 0\right)} .
$$

(ii) If $1-\prod_{j=1}^{m}\left(1-\mu_{i j}\right)^{w_{j}}=1-\prod_{j=1}^{m}\left(1-\mu_{i^{\prime} j}\right)^{w_{j}}$, then

$$
p_{i i^{\prime}}=\left\{\begin{array}{ll}
1, & \text { if } \prod_{j=1}^{m} v_{i j}^{w_{j}}<\prod_{j=1}^{m} v_{i^{\prime} j}^{w_{j}} \\
0, & \text { if } \prod_{j=1}^{m} v_{i j}^{w_{j}}>\prod_{j=1}^{m} v_{i^{\prime} j}^{w_{j}}
\end{array} .\right.
$$

It can be seen that the dominance degree of $Z_{i}$ over $Z_{i^{\prime}}$ can not be directly calculated using Eqs. (13) and (14) if $1-\prod_{j=1}^{m}\left(1-\mu_{i j}\right)^{w_{j}}<1-\prod_{j=1}^{m}\left(1-\mu_{i^{\prime} j}\right)^{w_{j}}$. For this situation, according to the study of $\mathrm{Yu}$ and $\mathrm{Xu}$ [46], the dominance degree of $Z_{i}$ over $Z_{i^{\prime}}$ can be calculated by

$$
p_{i i^{\prime}}=1-p_{i^{\prime} i} \text {. }
$$

In Eq. (15), $p_{i^{\prime} i}$ is the dominance degree of $Z_{i^{\prime}}$ over $Z_{i}$, which can be calculated by Eq. (13) 
since $1-\prod_{j=1}^{m}\left(1-\mu_{i^{\prime} j}\right)^{w_{j}}>1-\prod_{j=1}^{m}\left(1-\mu_{i j}\right)^{w_{j}}$.

Further, according to the obtained dominance degree $p_{i i^{\prime}}, i, i^{\prime}=1,2, \ldots, n$ and $i \neq i^{\prime}$, a dominance degree matrix on pairwise comparisons of alternative products can be constructed, i.e.,

$$
P=\left[p_{i i^{\prime}}\right]_{n \times n}=\begin{gathered}
A_{1} \\
A_{1} \\
A_{2} \\
\vdots \\
A_{n}
\end{gathered}\left[\begin{array}{cccc}
- & p_{12} & \cdots & A_{1 n} \\
p_{21} & - & \cdots & p_{2 n} \\
\vdots & \vdots & & \vdots \\
p_{n 1} & p_{n 2} & \cdots & -
\end{array}\right]
$$

where $p_{i i}="-"$ denotes that the dominance degree of $Z_{i}$ over itself is not considered, $i=1,2, \ldots, n$. According to $P=\left[p_{i i^{\prime}}\right]_{n \times n}$, the ranking of the alternative products can be determined using PROMETHEE II [47].

PROMETHEE II was first developed by Brans in 1982 [47] and has been widely applied to solve practical decision-making problems. Now, PROMETHEE II is regarded as one of the most well-known outranking methods. Usually, PROMETHEE II is consisted of the five parts [48-50]: (1) determining deviations based on pairwise comparisons of alternatives concerning each criterion; (2) constructing a relevant preference function for each criterion; (3) calculating global preference index; (4) calculating positive and negative outranking flows for each alternative; and (5) calculating the net outranking flow of each alternative and determining a complete ranking of alternatives. Compared with other outranking methods, PROMETHEE II has a clear logic and a simple computation process [51]. Further, since the obtained matrix $P=\left[p_{i i^{\prime}}\right]_{n \times n}$ is similar to the one used in PROMETHEE II, in this paper, PROMETHEE II is used to rank the alternative products.

Let $\Phi^{+}\left(A_{i}\right)$ be the dominance degree (or positive preference flow) which is a measure that alternative product $A_{i}$ is dominating the other alternative products, and $\Phi^{-}\left(A_{i}\right)$ be the non-dominance degree (or negative preference flow) which is a measure that alternative product $A_{i}$ is dominated by the other alternative products. $\Phi^{+}\left(A_{i}\right)$ and $\Phi^{-}\left(A_{i}\right)$ can be respectively calculated by the following Eqs. (16) and (17), i.e., 


$$
\begin{aligned}
& \Phi^{+}\left(A_{i}\right)=\frac{1}{n-1} \sum_{\substack{i^{\prime}=1 \\
i^{\prime} \neq 1}}^{n} p_{i i^{\prime}}, \quad i=1,2, \cdots, n, \\
& \Phi^{-}\left(A_{i}\right)=\frac{1}{n-1} \sum_{\substack{i^{\prime}=1 \\
i^{\prime} \neq i}}^{n} p_{i^{\prime} i}, \quad i=1,2, \cdots, n .
\end{aligned}
$$

Let $\Phi\left(A_{i}\right)$ be the relative dominance degree (or the net outranking flow) which measures the difference between dominance and non-dominance degrees of alternative product $A_{i}$. $\Phi\left(A_{i}\right)$ can be calculated by Eq. (18), i.e.,

$$
\Phi\left(A_{i}\right)=\Phi^{+}\left(A_{i}\right)-\Phi^{-}\left(A_{i}\right), \quad i=1,2, \ldots, n .
$$

$\Phi\left(A_{i}\right)$ can be regarded as the ranking value of alternative product $A_{i}$. Obviously, the greater $\Phi\left(A_{i}\right)$ is, the better alternative product $A_{i}$ will be. Therefore, according to net flows $\Phi\left(A_{1}\right), \Phi\left(A_{2}\right), \cdots, \Phi\left(A_{n}\right)$, a ranking of the alternative products can be determined.

In summary, the proposed process for ranking products through online reviews is given below.

Step 1. Crawl online review $D_{i k}=\left(D_{i k}^{1}, D_{i k}^{2}, \ldots, D_{i k}^{m}\right)$ from the related website using web crawler software, $i=1,2, \ldots, n, k=1,2, \ldots, q_{i}$. Then, determine the notional words of each review $\bar{W}_{i k}=\left(\bar{W}_{i k}^{1}, \bar{W}_{i k}^{2}, \ldots, \bar{W}_{i k}^{m}\right)$ by preprocessing the obtained online reviews using ICTCLAS 2015, $i=1,2, \ldots, n, k=1,2, \ldots, q_{i}$.

Step 2. Establish positive and negative sentiment dictionaries $\bar{W}_{j}^{+}$and $\bar{W}_{j}^{-}$concerning feature $f_{j}$ using Eqs. (1)-(4), $j=1,2, \ldots, m$.

Step 3. Identify the sentiment orientation of $D_{i k}^{j}$ (i.e., determine indicator vector $\left.S_{i k}^{j}=\left(\alpha_{i k}^{j}, \beta_{i k}^{j}, \gamma_{i k}^{j}\right)\right)$ using Algorithm 1, $i=1,2, \ldots, n, j=1,2, \ldots, m, k=1,2, \ldots, q_{i}$.

Step 4. Determine intuitionistic fuzzy number $x_{i j}=\left(\mu_{i j}, v_{i j}\right)$ of alternative product $A_{i}$ concerning feature $f_{j}$ using Eqs. (5)-(11), $i=1,2, \ldots, n, j=1,2, \ldots, m$.

Step 5. Calculate the overall intuitionistic fuzzy number $z_{i}$ of alternative product $A_{i}$ using Eq. (12), $i=1,2, \ldots, n$. 
Step 6. Construct dominance degree matrix $P=\left[p_{i i^{\prime}}\right]_{n \times n}$ using Eqs. (13)-(15). Then, calculate the dominance degree $\left(\Phi^{+}\left(A_{1}\right)\right)$, non-dominance degree $\left(\Phi^{-}\left(A_{1}\right)\right)$ and relative dominance degree $\left(\Phi\left(A_{i}\right)\right)$ of alternative product $A_{i}$ using Eqs. (16)-(18), $i=1,2, \ldots, n$. Further, determine a ranking of alternative products according to the obtained $\Phi\left(A_{i}\right)$, $i=1,2, \ldots, n$.

\section{Case study}

In this section, a case study on ranking the automobile products through online reviews is given to illustrate the use of the proposed method.

With the development of China's economy, automobiles gradually enter China's common families. More than 10 millions of consumers purchase new automobiles every year. However, since common consumers usually have limited knowledge and expertise on automobiles, it may be difficult for a common consumer to select a desirable one from the large number of automobiles of different brands. For this, several automobile review websites have been developed, which encourage consumers to post online reviews concerning the automobiles that they have bought, such as Automobile home (http://www.autohome.com.cn/) and PCauto (http://www.pcauto.com.cn/), etc. Consider a consumer wants to buy a SUV from four alternative SUVs. The four alternative SUVs are:

$A_{1}$ : Honda CR-V 2013, 2.4L (two-wheel drive Deluxe Edition);

$A_{2}$ : Mazda CX-5 2013, 2.5L (automatic four-wheel drive Deluxe Edition);

$A_{3}$ : Nissan X-Trail CVT 2014, 2.5L (Deluxe Edition);

$A_{4}$ : Compass 2014, 2.4L (four-wheel drive Comfort Edition).

To select a desirable SUV, the consumer concerns the eight features of SUVs, i.e., oil consumption $\left(f_{1}\right)$, comfort $\left(f_{2}\right)$, cost performance $\left(f_{3}\right)$, space $\left(f_{4}\right)$, power $\left(f_{5}\right)$, appearance $\left(f_{6}\right)$, controllability $\left(f_{7}\right)$, and car interior $\left(f_{8}\right)$. Meanwhile, the consumer provides the vector 
of weights of the eight features, i.e., $w=(0.2,0.2,0.1,0.1,0.1,0.1,0.1,0.1)$. To support the consumer's purchase decision, the method proposed in this paper is used to rank the four SUVs. According to the process shown in Fig. 3 and the steps given in Section 4, the computation processes and results are presented as below.

First of all, online reviews of the four SUVs concerning the eight features are crawled from Automobile home on August 3, 2015. In this case, we use Locoy Spider (http://www.locoy.com/) to crawl the online reviews, which is an online data collection software. In fact, other crawlers are also competent to obtain the online reviews, such as Bazhuayu (http://www.bazhuayu.com/), Kuangren (http://www.kuangren.cc/), and so on. The numbers of obtained reviews concerning different SUVs are 416, 412, 425 and 426, respectively. The obtained reviews can be expressed by $D_{i k}=\left(D_{i k}^{1}, D_{i k}^{2}, \ldots, D_{i k}^{8}\right)$, $i=1,2,3,4, k=1,2, \ldots, q_{i}, q_{1}=416, q_{2}=412, q_{3}=425$ and $q_{4}=426$, respectively. Then, by preprocessing the online reviews, the set of notional words of each review $D_{i k}$ concerning feature $f_{j}$ is determined, i.e., $\bar{W}_{i k}^{j}, i=1,2,3,4, k=1,2, \ldots, q_{i}, q_{1}=416$, $q_{2}=412, q_{3}=425$ and $q_{4}=426$. Take the alternative SUV $A_{1}$ as an example, the obtained sets of notional words of online reviews concerning $A_{1}$ are shown in Table 1, i.e., $\bar{W}_{1 k}^{1}, \bar{W}_{1 k}^{2}, \ldots, \bar{W}_{1 k}^{8}, \quad k=1,2, \cdots, 416$. Using Eqs. (1)-(4), positive sentiment dictionary $\bar{W}_{j}^{+}$and negative sentiment dictionary $\bar{W}_{j}^{-}$concerning feature $f_{j}$ are established, which are shown in Table 2, $j=1,2, \ldots, 8$.

\section{Table 1}

The sets of notional words of online reviews on SUV $A_{1}$ concerning each feature.

\begin{tabular}{|c|c|c|c|c|}
\hline \multirow{2}{*}{$\bar{W}_{1 k}$} & \multicolumn{4}{|l|}{ The online reviews on SUV $A_{1}$} \\
\hline & $k=1$ & $k=2$ & $\ldots$ & $k=416$ \\
\hline $\bar{W}_{1 k}^{1}$ & $\begin{array}{l}\text { 油耗不算大 (Fuel-consumption is } \\
\text { not big) } \Rightarrow \text { 油耗 } / \mathrm{n} \text { 不/d 算/v 大/a } \\
\text { (fuel-consumption /n, is /v, not /d, } \\
\text { big /a) }\end{array}$ & $\begin{array}{l}\text { 油耗相当低 (Fuel-consumption is } \\
\text { quite low) } \Rightarrow \text { 油耗 } / n \text { 相当/d 低/a } \\
\text { (fuel-consumption /n, quite /d, low /a) }\end{array}$ & $\cdots$ & $\vdots$ \\
\hline $\bar{W}_{1 k}^{2}$ & $\begin{array}{l}\text { 座椅很舒适 (The seat is very } \\
\text { conformable) } \Rightarrow \text { 座椅/n 很/d 舒适 } \\
\text { /a (seat /n, very /d, conformable /a) }\end{array}$ & $\begin{array}{l}\text { 隔音效果好 (Effect of sound } \\
\text { insulation is good) } \Rightarrow \text { 隔音/ } \mathrm{n} \text { 效果 } \\
/ \mathrm{n} \text { 好/a (sound-insulation } / \mathrm{n} \text {, effect } / \mathrm{n} \text {, }\end{array}$ & $\cdots$ & $\vdots$ \\
\hline
\end{tabular}


$\operatorname{good} / \mathrm{a})$

$\bar{W}_{1 k}^{8} \quad$ 内饰比较粗糙 (Interior is relatively rough) $\Rightarrow$ 内饰/ $\mathrm{n}$ 比较/d 粗糙/a (interior $/ \mathrm{n}$, relatively /d, rough /a)

仪表盘简洁 (The dashboard is $\cdots \quad$ : succinct) $\Rightarrow$ 仪表盘/n 简洁 /a (dashboard /n, succinct/a)

Note: n (noun); d (adverb); v (verb); a (adjective).

Using Algorithm 1, the sentiment orientation of $D_{i k}^{j}$ is identified, i.e., indicator vector $S_{i k}^{j}=\left(\alpha_{i k}^{j}, \beta_{i k}^{j}, \gamma_{i k}^{j}\right)$ is determined, $i=1,2,3,4, j=1,2, \ldots, 8, k=1,2, \ldots, q_{i}, \quad q_{1}=416$, $q_{2}=412, q_{3}=425$ and $q_{4}=426$. Take “油耗/n 不 $/ \mathrm{d}$ 算/v 大/a” (fuel-consumption /n, is /v, not /d, big /a), i.e., $D_{11}^{1}$, as an example. The notional words “油耗/n”, “不/d”, “算/v” and “大/a” are respectively compared with the words in $\bar{W}_{1}^{+}, \bar{W}_{1}^{-}$and $\bar{W}_{\text {neg }}$. There is no word matched with the words in $\bar{W}_{1}^{+}$, i.e., $\bar{W}_{1}^{+} \cap \bar{W}_{11}^{1}=\varnothing$, then according to lines 5-8 in Algorithm 1, we have $S_{11}^{1+} \leftarrow 0$; the “大” (big) is matched with the words in $\bar{W}_{1}^{-}$, i.e., $\bar{W}_{1}^{-} \cap \bar{W}_{11}^{1} \neq \varnothing$, then according to lines 9-12 in Algorithm 1, we have $S_{11}^{1-} \leftarrow 1$; the “不” (not) is matched with the words in $\bar{W}_{\text {neg }}$, i.e., $\bar{W}_{\text {neg }} \cap \bar{W}_{11}^{1} \neq \varnothing$, then according to lines 13-16 in Algorithm 1 , we have $S_{11}^{1 \text { neg }} \leftarrow 1$. Further, according to lines $19-22$ in Algorithm 1, we have $S_{11}^{1}=\left(\alpha_{11}^{1}, \beta_{11}^{1}, \gamma_{11}^{1}\right)=(1,0,0)$, i.e., the sentiment orientation of $D_{11}^{1}$ is positive.

\section{Table 2}

Positive and negative sentiment dictionaries $\bar{W}_{j}^{+}$and $\bar{W}_{j}^{-}$concerning each feature.

\begin{tabular}{lll}
\hline Features & Positive sentiment dictionary $\bar{W}_{j}^{+}$ & Negative sentiment dictionary $\bar{W}_{j}^{-}$ \\
\hline$f_{1}$ & $\begin{array}{l}\text { 满意 (satisfy), 低 (low), 好 (good), 省 } \\
\text { (save), .. }\end{array}$ & 高 (high), 差 (poor), 大 (big), .. \\
$f_{2}$ & $\begin{array}{l}\text { 舒适 (comfortable), 优秀 (excellent), 好 } \\
\text { (good), .. }\end{array}$ & $\begin{array}{l}\text { 差 (poor), 颠 (bumpy), 硬 (hard), .. } \\
\vdots\end{array}$ \\
$\vdots$ & $\begin{array}{l}\text { 精 致 (exquisite), 温 馨 } \\
\text { (luxury), (warm), 高 档 }\end{array}$ & $\begin{array}{l}\text { 离谱 (outrageous), 寒酸 (shabby), 粗鋉 } \\
\text { (crude), .. }\end{array}$ \\
\hline$f_{8}$ & &
\end{tabular}

\section{Table 3}

The values of $q_{i j}^{\text {pos }}, q_{i j}^{\text {neu }}$ and $q_{i j}^{\text {neg }}, i=1,2,3,4, j=1,2, \ldots, 8$. 


\begin{tabular}{|c|c|c|c|c|c|c|c|c|c|c|c|c|}
\hline & \multicolumn{3}{|c|}{$A_{1}$} & \multicolumn{3}{|c|}{$A_{2}$} & \multicolumn{3}{|c|}{$A_{3}$} & \multicolumn{3}{|c|}{$A_{4}$} \\
\hline & $q_{1 j}^{\text {pos }}$ & $q_{1 j}^{\text {neg }}$ & $q_{1 j}^{\text {neu }}$ & $q_{2 j}^{\text {pos }}$ & $q_{2 j}^{\mathrm{neg}}$ & $q_{2 j}^{\text {neu }}$ & $q_{3 j}^{\text {pos }}$ & $q_{3 j}^{\text {neg }}$ & $q_{3 j}^{\text {neu }}$ & $q_{4 j}^{\text {pos }}$ & $q_{4 j}^{\mathrm{neg}}$ & $q_{4 j}^{\text {neu }}$ \\
\hline$f_{1}$ & 557.7 & 145.6 & 61.96 & 440.8 & 132.1 & 39.2 & 357.8 & 56.2 & 18.9 & 354.8 & 103.8 & 73.4 \\
\hline$f_{2}$ & 500.6 & 59.5 & 56.9 & 350.6 & 139.9 & 82.7 & 378.0 & 6.1 & 3.9 & 329.2 & 102.6 & 36.7 \\
\hline$f_{3}$ & 513.7 & 77.9 & 16.7 & 548.8 & 26.8 & 2.37 & 327.1 & 61.6 & 1.3 & 352.0 & 75.5 & 13.7 \\
\hline$f_{4}$ & 597.3 & 5.4 & 1.3 & 409.4 & 106.3 & 61.9 & 377.3 & 4.3 & 6.3 & 276.3 & 88.4 & 111.2 \\
\hline$f_{5}$ & 414.9 & 107.1 & 33.5 & 521.5 & 38.9 & 17.6 & 363.3 & 22.3 & 4.4 & 430.6 & 32.0 & 8.5 \\
\hline$f_{6}$ & 510.8 & 81.6 & 18.3 & 574.7 & 2.1 & 1.1 & 332.4 & 41.3 & 21.2 & 471.4 & 1.7 & 1.6 \\
\hline$f_{7}$ & 387.9 & 166.2 & 49.8 & 5517 & 15.3 & 4.0 & 286.5 & 69.1 & 29.7 & 413.7 & 31.2 & 23.9 \\
\hline$f_{8}$ & 128.0 & 140.1 & 335.8 & 163.6 & 239.9 & 167.5 & 333.6 & 46.6 & 13.8 & 198.3 & 170.6 & 104.2 \\
\hline
\end{tabular}

Then, $q_{i j}^{\text {pos }}, q_{i j}^{\text {neu }}$ and $q_{i j}^{\text {neg }}$ are calculated using Eqs. (5)-(8), $i=1,2,3,4, j=1,2, \ldots, 8$, $k=1,2, \ldots, q_{i}, q_{1}=416, q_{2}=412, q_{3}=425$ and $q_{4}=426$. The values of $q_{i j}^{\text {pos }}, q_{i j}^{\text {neu }}$ and $q_{i j}^{\text {neg }}$ are shown in Table 3, $i=1,2,3,4, j=1,2, \ldots, 8$. Further, using Eqs. (9)-(11), the intuitionistic fuzzy number $x_{i j}=\left(\mu_{i j}, v_{i j}\right)$ of alternative SUV $A_{i}$ concerning feature $f_{j}$ is determined, $i=1,2,3,4, j=1,2, \ldots, 8$. The obtained intuitionistic fuzzy numbers are shown in Table 4.

\section{Table 4}

Intuitionistic fuzzy numbers of SUV $A_{i}$ concerning feature $f_{j}, i=1,2,3,4, j=1,2, \ldots, 8$.

\begin{tabular}{|c|c|c|c|c|}
\hline \multirow{2}{*}{ Features } & \multicolumn{4}{|c|}{ Alternative products } \\
\hline & $A_{1}$ & $A_{2}$ & $A_{3}$ & $A_{4}$ \\
\hline$f_{1}$ & $(0.7288,0.0810)$ & $(0.7201,0.0640)$ & $(0.8265,0.0436)$ & $(0.6669,0.1380)$ \\
\hline$f_{2}$ & $(0.8113,0.0923)$ & $(0.6117,0.1443)$ & $(0.9742,0.0100)$ & $(0.7027,0.0784)$ \\
\hline$f_{3}$ & $(0.8445,0.0274)$ & $(0.9495,0.0041)$ & $(0.8387,0.0033)$ & $(0.7979,0.0310)$ \\
\hline$f_{4}$ & $(0.9891,0.0020)$ & $(0.7088,0.1072)$ & $(0.9725,0.0163)$ & $(0.5807,0.2336)$ \\
\hline$f_{5}$ & $(0.7470,0.0603)$ & $(0.9023,0.0304)$ & $(0.9314,0.0114)$ & $(0.9139,0.0181)$ \\
\hline$f_{6}$ & $(0.8364,0.0299)$ & $(0.9944,0.0019)$ & $(0.8419,0.0536)$ & $(0.9965,0.0101)$ \\
\hline$f_{7}$ & $(0.6424,0.0824)$ & $(0.9662,0.0070)$ & $(0.7436,0.0772)$ & $(0.8825,0.0509)$ \\
\hline$f_{8}$ & $(0.2120,0.5560)$ & $(0.2866,0.2934)$ & $(0.8465,0.0351)$ & $(0.4191,0.2203)$ \\
\hline
\end{tabular}

Using Eq. (12), the overall intuitionistic fuzzy number $Z_{i}$ of alternative SUV $A_{i}$ is calculated, i.e., $z_{1}=(0.8132,0.0550), \quad z_{2}=(0.8633,0.0368), z_{3}=(0.9093,0.0211)$ and $Z_{4}=(0.8326,0.0667)$. Using Eqs. (13)-(15), dominance degree $p_{i i^{\prime}}$ is calculated, 
$i, i^{\prime}=1,2,3,4$, and dominance degree matrix $P=\left[p_{i i^{\prime}}\right]_{4 \times 4}$ can be constructed, i.e.,

$$
\begin{gathered}
A_{1} \\
P=\frac{A_{2}}{A_{2}} \\
A_{1} \\
A_{3} \\
A_{4}
\end{gathered}\left[\begin{array}{cccc}
- & 0 & 0 & A_{4} \\
1 & - & 0 & 1 \\
1 & 1 & - & 1 \\
0.623 & 0 & 0 & -
\end{array}\right] .
$$

Based on matrix $P$, dominance degree $\left(\Phi^{+}\left(A_{i}\right)\right)$, non-dominance degree $\left(\Phi^{-}\left(A_{i}\right)\right)$ and relative dominance degree ( $\left.\Phi\left(A_{i}\right)\right)$ of each SUV can be calculated using Eqs. (16)-(18). The values of $\Phi^{+}\left(A_{i}\right), \Phi^{-}\left(A_{i}\right)$ and $\Phi\left(A_{i}\right)$ are shown in Table 5, $i=1,2,3,4$. According to the obtained $\Phi\left(A_{i}\right)$, a ranking of the four alternative SUVs can be determined, i.e., $A_{3} \succ A_{2} \succ A_{4} \succ A_{1}$. The ranking result is presented to the consumer to support the consumer to select a desirable SUV.

Table 5

The values of $\Phi^{+}\left(A_{i}\right), \Phi^{-}\left(A_{i}\right)$ and $\Phi\left(A_{i}\right), \quad i=1,2,3,4$.

\begin{tabular}{llll}
\hline$A_{i}$ & $\Phi^{+}\left(A_{i}\right)$ & $\Phi^{-}\left(A_{i}\right)$ & $\Phi\left(A_{i}\right)$ \\
\hline$A_{1}$ & 0.1256 & 0.8744 & -0.7488 \\
$A_{2}$ & 0.6667 & 0.3333 & 0.3334 \\
$A_{3}$ & 1.0000 & 0.0000 & 1.0000 \\
$A_{4}$ & 0.2077 & 0.7923 & -0.5846 \\
\hline
\end{tabular}

To investigate the characteristics and advantages of the proposed method, the comparisons and experiments need to be further conducted. It is necessary to point out that, although the existing methods [6-11] can be used to determine the rankings of products based on online reviews, there are some differences between the problem considered in our study and those considered in the existing studies [6-11]. The main difference is reflected in the aspect of the consideration of product features and feature weights. The problem considered in the existing studies [6-11] is how to rank similar products from the perspective of a third party. Thus, in the existing studies, the product features and feature weights are not considered $[6,8,10]$ or are objectively determined according to the online reviews $[7,9,11]$. But, the problem considered in our study is how to support the consumers' purchase decisions. Thus, in our 
study, the product features and feature weights are subjectively determined according to the consumer's preference. Usually, different methods could be comparable only if they are used to solve the same problem. For this, a special case of equal feature weights is considered, i.e., $w_{1}=w_{2}=\ldots=w_{8}=0.125$. The special case of equal feature weights can be regarded as approximately equivalent to the situations that product features of online reviews are not considered. Under the special case of equal feature weights, the proposed method and the methods proposed in literatures $[6,8,11]$ are simultaneously used to determine the rankings of the four alternative SUVs. The same ranking result is obtained using the method proposed by Zhang et al. [8], the method proposed by Najmi et al. [11] and the method proposed in this paper, i.e., $A_{3} \succ A_{2} \succ A_{4} \succ A_{1}$. The ranking result obtained by the method proposed by Zhang et al. [6] is $A_{3} \succ A_{2} \succ A_{1} \succ A_{4}$. It is necessary to further illustrate that the methods proposed in literatures $[7,9,10]$ are not used since additional expert evaluations are needed in literature [9] and multiple ranking results of alternatives are simultaneously obtained in literatures [7, 10]. It can be seen that, under the special case of equal feature weights, same or similar ranking results can be obtained by the proposed method and the methods proposed in literatures $[6,8,11]$. However, if the different product features or feature weights are considered, then existing methods can not be used to obtain the ranking results of alternative products since subjective product features and feature weights are not considered in the existing studies [6-11]. Table 6 shows the ranking results of the alternative SUVs obtained by the proposed method, where different feature weights are considered. It can be seen from Table 6 that, by the proposed method, different ranking results could be obtained if different product features or feature weights are considered. It means that, by the proposed method, different products could be recommended to the consumers with different subjective preference on product features. Moreover, an experiment with different numbers of alternative products is conducted to observe the consistency of the ranking results of alternative products obtained by the proposed method. For this, other four alternative SUVs are added, they are Dongfeng Peugeot 3008 2015, 1.6THP $\left(A_{5}\right)$, Mitsubishi Outlander 2016, 2.4L $\left(A_{6}\right)$, Faw Toyota RAV 2015, 2.0CVT $\left(A_{7}\right)$ and Tiguan 2015, 1.8TSI $\left(A_{8}\right)$. The reviews concerning the added alternative SUVs are also crawled from Automobile home. Ranking results with 
different numbers of alternative SUVs are $A_{3} \succ A_{5} \succ A_{2} \succ A_{4} \succ A_{1}, \quad A_{3} \succ A_{5} \succ A_{2} \succ$ $A_{6} \succ A_{4} \succ A_{1}, \quad A_{3} \succ A_{5} \succ A_{2} \succ A_{7} \succ A_{6} \succ A_{4} \succ A_{1}$ and $A_{3} \succ A_{5} \succ A_{2} \succ A_{7} \succ A_{6} \succ A_{4} \succ A_{8}$ $\succ A_{1}$. It can be seen that the relative superiority relationships among the original alternative SUVs $\left(A_{1}, A_{2}, A_{3}\right.$ and $\left.A_{4}\right)$ are not changed with the addition of new alternative SUVs $\left(A_{5}, A_{6}\right.$, $A_{7}$ and $\left.A_{8}\right)$.

Table 6

Ranking results of alternative SUVs with different feature weights.

\begin{tabular}{|c|c|c|c|c|c|c|c|c|}
\hline \multirow{2}{*}{$\begin{array}{l}\text { Ranking results of } \\
\text { alternative products }\end{array}$} & \multicolumn{8}{|c|}{ Feature weights } \\
\hline & $w_{1}$ & $w_{2}$ & $w_{3}$ & $w_{4}$ & $w_{5}$ & $w_{6}$ & $w_{7}$ & $w_{8}$ \\
\hline$A_{3} \succ A_{2} \succ A_{4} \succ A_{1}$ & 0.125 & 0.125 & 0.125 & 0.125 & 0.125 & 0.125 & 0.125 & 0.125 \\
\hline$A_{2} \succ A_{3} \succ A_{4} \succ A_{1}$ & 0.1 & 0.1 & 0.1 & 0.1 & 0.1 & 0.1 & 0.3 & 0.1 \\
\hline$A_{2} \succ A_{4} \succ A_{3} \succ A_{1}$ & 0.1 & 0.1 & 0.1 & 0.1 & 0.1 & 0.3 & 0.1 & 0.1 \\
\hline$A_{3} \succ A_{1} \succ A_{2} \succ A_{4}$ & 0.1 & 0.1 & 0.1 & 0.3 & 0.1 & 0.1 & 0.1 & 0.1 \\
\hline$A_{1} \succ A_{3} \succ A_{2} \succ A_{4}$ & 0.1 & 0.2 & 0.1 & 0.5 & 0 & 0 & 0.1 & 0 \\
\hline$A_{4} \succ A_{2} \succ A_{3} \succ A_{1}$ & 0 & 0.3 & 0 & 0 & 0.1 & 0.5 & 0 & 0.1 \\
\hline
\end{tabular}

\section{Conclusions}

This paper presents a novel method based on the sentiment analysis technique and the intuitionistic fuzzy set theory to rank the alternative products through online reviews. In the method, online reviews of the alternative products concerning the features are crawled using the crawler software. Positive and negative sentiment dictionaries with respect to each product feature are established according to the crawled online reviews and the HowNet sentiment dictionary. Using the developed algorithm, the positive, neutral or negative sentiment orientation on the product concerning each product feature in each review is identified, and the weighted percentages of reviews with positive, neutral and negative sentiment orientations in all the reviews of one product concerning one feature are calculated. According to the obtained weighted percentages, an intuitionistic fuzzy number is formed based on intuitionistic fuzzy set theory for representing the performance of the alternative product concerning the feature, and an overall intuitionistic fuzzy number of each alternative product is calculated using the IFWA operator. Further, the dominance degrees on pairwise comparisons of the alternative products are calculated, and a ranking of the alternative products is determined using the PROMETHEE II method. The major contributions of this paper are discussed as follows. 
First, to support consumers' purchase decisions, this paper formulates a new problem on ranking products through online reviews. In the problem, the alternative products, product features and weights of product features are determined according to the subjective preference of the consumer. The problem has a lot of practical backgrounds and needs to be paid more attentions.

Second, this paper presents a resolution process for solving the problem. The process includes the two parts: identifying sentiment orientations of the online reviews based on sentiment analysis technique, and ranking the alternative products based on the intuitionistic fuzzy set theory. The resolution process lays a good foundation for further conducting studies on ranking products through online reviews.

Third, for identifying the positive, neutral and negative sentiment orientations of online reviews, a new algorithm based on sentiment dictionaries is proposed. The algorithm has a clear logic and a simple operational process, which is a valuable attempt for refining more valuable information for ranking products through online reviews.

Fourth, a key strength of the proposed method is that the identified positive, neutral and negative sentiment orientations on an alternative product concerning a feature are converted into an intuitionistic fuzzy number for representing the performance of the product concerning the feature. Based on intuitionistic fuzzy set theory, a large number of sentiment orientations of online reviews on an alternative product concerning a product feature can be simply and completely represented by an intuitionistic fuzzy number. It is a new idea for processing and fusing a large number of sentiment orientations embedded in the online reviews.

It is important to highlight that, the proposed method has a clear logic and a simple computation process. It is also important for developing and enriching theories and methods for ranking products through online reviews.

In terms of future research, to support consumers to use the proposed method to make purchase decision more conveniently, the support system based on the proposed method needs to be developed. Besides, the situation that one or more alternatives are new products with few online reviews needs to be considered.

\section{Acknowledgement}

This work was partly supported by the National Science Foundation of China (Project Nos. 71371002 and 71571039), the Fundamental Research Funds for the Central Universities, China (Project No. N140607001), and the 111 Project (B16009). 


\section{References}

[1] H. Chen, R. Chiang, V. Storey, Business intelligence and analytics: from big data to big impact, MIS Q. 36 (4) (2012) 1165-1188.

[2] Z. Daniel, C. Hsinchun, R. Lusch, L. Shu-Hsing, Social media analytics and intelligence, IEEE Intell. Syst. 25 (2010) 13-16.

[3] B. Bickart, R.M. Schindler, Internet forums as influential sources of consumer information, J. Interact. Market. 15 (2001) 31-40.

[4] T. Hennig-Thurau, K.P. Gwinner, G. Walsh, D.D. Gremler, Electronic word-of-mouth via consumer-opinion platforms: what motivates consumers to articulate themselves on Internet? J. Interact. Market. 18 (2004) 38-52.

[5] Z.J. Zha, J. Yu, J. Tang, M. Wang, T.S. Chua, Product aspect ranking and its applications, IEEE T. Knowl. Data En. 26(5) (2014) 1211-1224.

[6] K. Zhang, R. Narayanan, A. Choudhary, Mining online customer reviews for ranking products, Technical Report, EECS Department, Northwestern University, 2009.

[7] K. Zhang, R. Narayanan, A. Choudhary, Voice of the customers: Mining online customer reviews for product feature-based ranking, In: WOSN'10, Boston, USA, 2010.

[8] K. Zhang, Y. Cheng, W.K. Liao, A. Choudhary, Mining millions of reviews: a technique to rank products based on importance of reviews, Proceedings of the 13th International Conference on Electronic Commerce, ACM, New York, USA, 2011.

[9] Y. Peng, G. Kou, J. Li, A fuzzy PROMETHEE approach for mining customer reviews in Chinese, Arab. J. Sci. Eng. 39 (6) (2014) 5245-5252.

[10]K. Chen, G. Kou, J. Shang, Y. Chen, Visualizing market structure through online product reviews: Integrate topic modeling, TOPSIS, and multi-dimensional scaling approaches, Electron. Commer. Res. Appl. 14 (2015) 58-74.

[11]E. Najmi, K. Hashmi, Z. Malik, A. Rezgui, H.U. Khan, CAPRA: A comprehensive approach to product ranking using customer reviews, Computing, 97 (8) (2015) 843-866.

[12]Z.S. Xu, Intuitionistic fuzzy aggregation operators, IEEE Trans. Fuzzy Syst. 15 (6) (2007) 1179-1187.

[13]R.M. Rodriguez, L. Martínez, F. Herrera, Hesitant fuzzy linguistic term sets for decision making, IEEE Trans. Fuzzy Syst. 20 (2012) 109-119.

[14]F. Herrera, L. Martínez, V. Torra, Z.S. Xu, Hesitant fuzzy sets: an emerging tool in decision making, Int. J. Intell. Syst. 29 (6) (2014) 493-944.

[15]K.T. Atanassov, Intuitionistic fuzzy sets, Fuzzy Sets Syst. 20 (1) (1986) 87-96. 
[16]Z.S. Xu, N. Zhao, Information fusion for intuitionistic fuzzy decision making: An overview, Inform. Fusion 28 (2016) 10-23.

[17]S.L. Huang, W.C. Cheng, Discovering Chinese sentence patterns for feature-based opinion summarization, Electron. Commer. Res. Appl. 14 (2015) 582-591.

[18]W. Medhat, A. Hassan, H. Korashy, Sentiment analysis algorithms and applications: A survey, Ain Shams Eng. J. 5 (2014) 1093-1113.

[19]K. Ravi, V. Ravi, A survey on opinion mining and sentiment analysis: Tasks, approaches and applications, Knowl.-Based Syst. 89 (2015) 14-46.

[20]J. Serrano-Guerrero, J.A. Olivas, F.P. Romero, E Herrera-Viedma, Sentiment analysis: A review and comparative analysis of web services, Inform. Sci. 311 (2015) 18-38.

[21]J. A. Balazs, J. D. Velásquez, Opinion Mining and Information Fusion: A survey, Inform. Fusion 27 (2016) 95-110.

[22]J. C. Bertot, P. T. Jaeger, D. Hansen, The impact of polices on government social media usage: Issues, challenges, and recommendations, Gov. Inform. Q. 29(1) (2012), 30-40.

[23]O. Netzer, R. Feldman, J. Goldenberg, M. Fresko, Mine your own business: Market-structure surveillance through text mining, Market. Sci. 31 (3) (2012), 521-543.

[24]E. Marrese-Taylor, J.D. Velásquez, F. Bravo-Marquez, A novel deterministic approach for aspect-based opinion mining in tourism products reviews, Expert Syst. Appl. 41 (2012) 7764-7775.

[25]W.H. Zhang, H. Xu, W. Wan, Weakness Finder: Find product weakness from Chinese reviews by using aspects based sentiment analysis, Expert Syst. Appl. 39 (11) (2012) 10283-10291.

[26]M. Tsytsarau, T. Palpanas, Survey on mining subjective data on the web, Data Min. Knowl. Discov. 24 (2012) 478-514.

[27]F.H. Khan, U. Qamar, S. Bashir, eSAP: A decision support framework for enhanced sentiment analysis and polarity classification, Inform. Sci. 367-368 (2016) 862-873.

[28]X. Bai, Predicting consumer sentiments from online text, Decis. Support Syst. 50 (2011) 732-42.

[29]H. Kang, S.J. Yoo, D. Han, Senti-lexicon and improved Naïve Bayes algorithms for sentiment analysis of restaurant reviews, Expert Syst. Appl. 39 (2012) 6000-6010.

[30]A. Duric, F. Song, Feature selection for sentiment analysis based on content and syntax models, Decis. Support Syst. 53 (2012) 704-711.

[31]F. Tian, F. Wu, K.M. Chao, Q.H. Zheng, N. Shahc, T. Lan, J. Yue, A topic sentence-based instance transfer method for imbalanced sentiment classification of Chinese product 
reviews, Electron. Commer. Res. Appl. 16 (2016) 66-76.

[32]D.W. Zhang, H. Xu, Z.C. Su, Y.F. Xu, Chinese comments sentiment classification based on word2vec and SVM ${ }^{\text {perf }}$, Expert Syst. Appl. 42 (4) (2015) 1857-1863.

[33]Y. Li, Q. Ye, Z. Zhang, T. Wang, Snippet-based unsupervised approach for sentiment classification of Chinese online reviews. Int. J. Inf. Technol. Decis. Making, 10 (6) (2011), 1097-1110.

[34]P. Turney, Thumbs up or thumbs down?: semantic orientation applied to unsupervised classification of reviews, In: Proceedings of annual meeting of the Association for Computational Linguistics (2002).

[35]F.H. Khan, S. Bashir, U. Qamar, TOM: twitter opinion mining framework using hybrid classification scheme, Decis. Support Syst. 57 (2014) 245-257.

[36]F. H. Khan, U. Qamar, S. Bashir, SWIMS: Semi-supervised subjective feature weighting and intelligent model selection for sentiment analysis, Knowl.-Based Syst. 100 (2016), 97-111.

[37]F.H. Khan, U. Qamar, S. Bashir, Building normalized SentiMI to enhance semi-supervised sentiment analysis, J. Intell. Fuzzy Syst. 29 (2015) 1805-1816.

[38]M. Hu, B. Liu, Mining and summarizing customer reviews. In: Proceedings of ACM SIGKDD international conference on Knowledge Discovery and Data Mining (2004).

[39]S. Kim, E. Hovy, Determining the sentiment of opinions. In: Proceedings of interntional conference on Computational Linguistics (2004).

[40] A. Moreo, M. Romero, J.L. Castro, J.M. Zurita, Lexicon-based comments-oriented news sentiment analyzer system, Expert Syst. Appl. 39 (10) (2012) 9166-9180.

[41]K. Xu, S.S. Liao, J. Li, Y. Song, Mining comparative opinions from customer reviews for competitive intelligence. Decis. Support Syst. 50 (2011) 743-754.

[42]T.L. Saaty, The Analytical Hierarchy Process, McGraw-Hill, Toronto, 1980.

[43]H.Q. Zhang, A. Sekhari, Y. Ouzrout, A. Bouras, Jointly identifying opinion mining elements and fuzzy measurement of opinion intensity to analyze product features, Eng. Appl. Artif. Intell. 47 (2016) 122-139.

[44]G. Qiu, B. Liu, J. Bu, C. Chen, Opinion word expansion and target extraction through double propagation, Comput. Linguist. 37 (1) (2011) 9-27.

[45]A. Kar, D.P. Mandal, Finding opinion strength using fuzzy logic on web reviews, Int. J. Eng. Ind. 2 (1) (2011) 37-43. 
[46]X.H. Yu, Z.S. Xu, Prioritized intuitionistic fuzzy aggregation operators, Inform. Fusion 14 (1) (2013) 108-116.

[47]J.P. Brans, L’ingénièrie de la décision; Elaboration d’instruments d’aide à la décision. La methode PROMETHEE, in: R. Nadeau, M. Landry (Eds.), L’aide à la décision: Nature, Instruments et Perspectives d'Avenir, Presses de l’Université Laval, Québec, Canada, 1982, 183-213.

[48]M. Behzadian, R.B. Kazemzadeh, A. Albadvi, M. Aghdasi, PROMETHEE: a comprehensive literature review on methodologies and applications, Eur. J. Oper. Res. 200(1) (2010) 198-215.

[49]J.P. Brans, P.H. Vincke, A preference ranking organization method (the PROMETHEE method for multiple criteria decision making), Manage. Sci. 31 (6) (1985) 647-656.

[50]T.Y. Chen. An interval type-2 fuzzy PROMETHEE method using a likelihood-based outranking comparison approach, Inform. Fusion 25 (2015) 105-120.

[51]J.P. Brans, P.H. Vincke, B. Mareschal, How to select and how to rank projects: the PROMETHEE method, Eur. J. Oper. Res. 24 (2) (1986) 228-238. 